\title{
Association of Race and Neighborhood Disadvantage with Patient Engagement in a Home-Based COVID-19 Remote Monitoring Program
}

\author{
Bradley A. Fritz, MD, MSc ${ }^{1,2}$ (D), Brett Ramsey, MBA ${ }^{7}$, Dick Taylor, $\mathrm{MD}^{3}$, \\ John Paul Shoup, MD4, Jennifer M. Schmidt, MD ${ }^{5}$, Megan Guinn, MBA, BSN, RN 4 , and \\ Thomas M. Maddox, MD, MSc $c^{1,6}$
}

\begin{abstract}
'Healthcare Innovation Lab, BJC HealthCare/Washington University School of Medicine, St. Louis, MO, USA; ${ }^{2}$ Department of Anesthesiology, Washington University School of Medicine, St. Louis, MO, USA; ${ }^{3}$ Epic 1, BJC HealthCare/Washington University School of Medicine, St. Louis, MO, USA; ${ }^{4}$ BJC Medical Group, St. Louis, MO, USA; ${ }^{5}$ Division of General Internal Medicine, Department of Medicine, Washington University School of Medicine, St. Louis, MO, USA; 'Division of Cardiology, Department of Medicine, Washington University School of Medicine, St. Louis, MO, USA
\end{abstract}

BACKGROUND: COVID-positive outpatients may benefit from remote monitoring, but such a program often relies on smartphone apps. This may introduce racial and socio-economic barriers to participation. Offering multiple methods for participation may address these barriers. OBJECTIVES: (1) To examine associations of race and neighborhood disadvantage with patient retention in a monitoring program offering two participation methods. (2) To measure the association of the program with emergency department visits and hospital admissions.

DESIGN: Retrospective propensity-matched cohort study.

PARTICIPANTS: COVID-positive outpatients at a single university-affiliated healthcare system and propensitymatched controls.

INTERVENTIONS: A home monitoring program providing daily symptom tracking via patient portal app or telephone calls.

MAIN MEASURES: Among program enrollees, retention (until 14 days, symptom resolution, or hospital admission) by race and neighborhood disadvantage, with stratification by program arm. In enrollees versus matched controls, emergency department utilization and hospital admission within 30 days.

KEY RESULTS: There were 7592 enrolled patients and 9710 matched controls. Black enrollees chose the telephone arm more frequently than White enrollees $168 \%$ versus $44 \%, p=0.009$ ), as did those from more versus less disadvantaged neighborhoods (59\% versus 43\%, $p=$ 0.02). Retention was similar in Black enrollees and White enrollees (63\% versus 62\%, $p=0.76$ ) and in more versus less disadvantaged neighborhoods $(63 \%$ versus $62 \%, p=$ 0.44). When stratified by program arm, Black enrollees had lower retention than White enrollees in the app arm (49\% versus $55 \%, p=0.01$ ), but not in the telephone arm (69\% versus $71 \%, p=0.12)$. Compared to controls, enrollees more frequently visited the emergency department (HR 1.71 [95\% CI 1.56-1.87]) and were admitted to the hospital (HR 1.16 [95\% CI 1.02-1.31]).

Prior Presentations: None.

Received May 11, 2021

Accepted October 8, 2021

Published online January 6, 2022
CONCLUSIONS: In a COVID-19 remote patient monitoring program, Black enrollees preferentially selected, and had higher retention in, telephone- over app-based monitoring. As a result, overall retention was similar between races. Remote monitoring programs with multiple modes may reduce barriers to participation.

KEY WORDS: ambulatory monitoring; COVID-19; race factors; facilities and services utilization.

J Gen Intern Med 37(4):838-46

DOI: $10.1007 / \mathrm{s} 11606-021-07207-4$

() Society of General Internal Medicine 2021

\section{INTRODUCTION}

Infections caused by SARS-CoV-2 have caused a pandemic that has pushed healthcare systems worldwide to their limits. Fortunately, most patients present with mild symptoms that can be managed via home quarantine. ${ }^{1,2}$ Patients require careful monitoring during their quarantine, because deterioration requiring hospitalization may occur after a period of apparent stability. ${ }^{3,4}$ Remote monitoring at home is an attractive management strategy because it reduces transmission risks associated with in-person follow-up ${ }^{5,6}$ and because it eliminates transportation barriers for economically disadvantaged or geo-

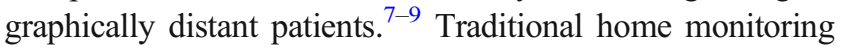
programs are labor-intensive, requiring nurses to call patients daily to assess symptoms. ${ }^{10}$ Emerging technologies have enabled automation of this process via smartphone apps, engaging staff only when medical intervention might be warranted. $^{11,12}$

Although automated home monitoring programs are attractive due to their scalability, these programs may create barriers to patient engagement. Patients must have access to a smart device and a reliable internet connection to participate. The well-described "digital divide" may prevent Black, economically disadvantaged, or older patients from participating in an app-based remote monitoring program. ${ }^{13-16}$ Accordingly, we designed a program that allowed for either app-based or 
telephone-based engagement options, with the goal to enable patients from all racial and economic groups to remain engaged. It was also necessary to understand the impact of the program on emergency department (ED) and hospital utilization because these facilities had become flooded with patients during the pandemic. ${ }^{5}$ It was unclear whether the program would decrease ED and hospital utilization due to earlier treatment of deteriorating patients in virtual settings or if it would increase utilization due to increased surveillance. Thus, the specific aims of this report were (1) to examine the associations of race and neighborhood economic disadvantage with patient retention in our program and (2) to measure the association of the program with ED visits and hospital admissions.

\section{METHODS}

\section{Study Design and Administration}

The remote monitoring program was implemented as a quality improvement project at BJC Healthcare and Washington University School of Medicine, a large university-affiliated healthcare system based in St. Louis, Missouri. A multidisciplinary team of clinicians, including internal medicine, pulmonology, and infectious disease specialists, refined the care pathway used in the program. This pathway was modeled on the program developed by the Cleveland Clinic using Epic's Care Companion Home Monitoring Program app. ${ }^{17,18}$ In parallel, an operational team allocated resources for patient monitoring and configured the Care Companion platform to accommodate the desired workflows in conjunction with analysts from Epic electronic health record (EHR). The first patient was enrolled on 4/6/2020. This manuscript presents a retrospective propensity-matched cohort study of patients who enrolled in the home monitoring program and COVIDpositive outpatients who did not enroll in the program. The Human Research Protection Office at Washington University School of Medicine approved this cohort study (\#202,103,247) and granted a waiver of informed consent to obtain data from the EHR. This report was written in compliance with STROBE guidelines. ${ }^{19}$

\section{Patient Population}

This study includes all patients who enrolled in the home monitoring program between 4/6/2020 and 12/7/2020. Patients were enrolled either by outreach from program staff or by their healthcare provider offering the program to them. Inclusion criteria for outreach from program staff included (1) a positive COVID-19 lab result in the past 14 days, (2) "confirmed COVID-19 infection" added to the EHR problem list within the past 30 days, or (3) an active COVID-19 isolation flag in the EHR. Exclusion criteria included age < 18 or inability to converse in English. Eligible patients with active Epic MyChart accounts received automated invitations in English to enroll through the app. Eligible patients without active MyChart accounts or who did not respond were enrolled by telephone.

Matched controls were selected from the group of adult patients residing in Missouri or Illinois with a positive COVID test performed in an outpatient setting in the BJC network between 4/1/2020 and 12/7/2020. Because the controls were limited to outpatients, any enrolled patients whose initial COVID test had been performed in an inpatient setting were excluded from the propensity-matched analyses.

\section{Intervention}

Each morning, patients completed a structured questionnaire via the Care Companion app or via telephone assessing appetite, cough, diarrhea, fever, shortness of breath, vomiting, and weakness (Supplement Figure S1). Temperature and oxygen saturation were also reported if the patient could measure them. Patients could choose between the app and telephone calls at the time of enrollment. Patients in the app arm who did not complete the questionnaire received an outbound call from a medical assistant to collect responses. If the patient's symptoms or self-reported vital signs fell outside pre-specified parameters (Supplement Table S1), then the case was escalated to a triage nurse. The nurse phoned the patient the same day to assess further and either advised the patient to self-monitor, arranged a video visit with a physician or an in-person respiratory clinic visit, or instructed the patient to seek emergency medical care. Daily symptom monitoring continued until symptom resolution, or for 14 days if the patient remained asymptomatic. Patients were automatically removed from the program if they were admitted to a hospital or if they could not be contacted for three consecutive days. In addition, patients could opt out at any time. Daily symptom questionnaire responses and notes documenting all phone calls were stored in the EHR.

\section{Variable Definitions}

Among the enrollees, key exposure variables included race and neighborhood disadvantage. Race was extracted from the EHR. The degree of neighborhood disadvantage was estimated using the Area Deprivation Index (ADI) score associated with the patients' zip codes. ${ }^{20}$ Patients residing in zip codes with an ADI score greater than the $6^{\text {th }}$ nationwide decile (which was the median value in this dataset) were classified as experiencing neighborhood disadvantage.

Among enrollees, the primary outcome metric compared across race and neighborhood disadvantage groups was retention in the program. Retention was defined as the percentage of enrollees who remained in the program until symptom resolution (or until 14 days if asymptomatic) or until hospital admission.

Among enrollees and matched controls, the outcome metrics were emergency department visits and hospital admissions. Encounters at any of the 12 BJC locations in Missouri 
or Illinois that use the Epic EHR were included. Each ED visit or admission was classified as potentially COVID-related if the primary diagnosis and/or primary problem associated with encounter contained any of the following text strings: anosmia, asthma, bronch*, COPD, corona*, cough, COVID, dyspnea, emesis, fatigue, febrile, fever, flu, hypox*, leukocytosis, mental status, myalgia, nausea, pneumonia, pulmonary, respiratory, sepsis, septic, shortness of breath, smell, SOA, SOB, URI, viral, virus, vomit, weak. For program enrollees, each ED visit or admission was further classified by whether the patient's case had been escalated to a home monitoring triage nurse during program enrollment.

\section{Analyses}

Analyses were conducted in R version 4.0.03 (R, Vienna, Austria). All available data were used, so the sample size was determined by the number of patients who had enrolled in the home monitoring program (with no sample size calculation). Demographic characteristics of the population were compared across race and neighborhood disadvantage strata using Pearson's chi-squared test (categorical variables), Fisher's exact test (race), or the Wilcoxon rank sum test (age). Patient zip codes were used to classify each patient as living either in the urban core of the city (Rural-Urban Commuting Area (RUCA) code equal to 1) or not. ${ }^{21}$ Program arm selection (telephone versus app) and retention were compared across race and neighborhood disadvantage strata using a chi-squared test. Comparisons across race strata were limited to White patients and Black patients due to the small number of patients reporting other races.

For the matched analysis, enrollees (excluding those initially diagnosed with COVID as an inpatient) were matched 1:2 to control patients based on the propensity score for enrollment. Propensity scores were calculated using a logistic regression adjusting for age, sex, ethnicity, residence in the urban core, diabetes, hypertension, coronary artery disease, heart failure, arrhythmia, chronic lung disease, chronic kidney disease, chronic liver disease, hypothyroidism, depression, and any cancer. Satisfactory matches had propensity scores within a caliper of 0.1 standard deviation, plus exact matches for race and neighborhood disadvantage. Matching was performed using the MatchIt package. ${ }^{22}$ Time from positive coronavirus test to ED visit and to hospital admission was compared between the matched groups in a Cox proportional hazards model. Patients were right-censored in the ED visit analysis if they were directly admitted to a hospital. The Cox model was repeated within each race and neighborhood disadvantage stratum, and within each program arm. Statistical significance was determined using $\alpha=0.05$.

\section{RESULTS}

\section{Patient Population}

Between 4/6/2020 and 12/7/2020, 7592 patients enrolled in the program (Fig. 1). The enrollees included 5083 White patients (67\%), 2160 Black patients (28\%), and 349 of other or unknown race $(5 \%)$. Black enrollees were younger than White enrollees, more likely to be female, and more likely to live in disadvantaged neighborhoods or in the urban core (Table 1). Of the 7465 enrollees whose addresses could be mapped to an ADI score, 3585 (48\%) lived in more disadvantaged neighborhoods, while 3880 (52\%) lived in less disadvantaged neighborhoods. Those from more disadvantaged neighborhoods were slightly younger and less likely to live in the urban core but had a similar sex distribution (Table 1).

Compared to White enrollees, Black enrollees were more likely to select the telephone arm $(68 \%$ versus $44 \%, p=$

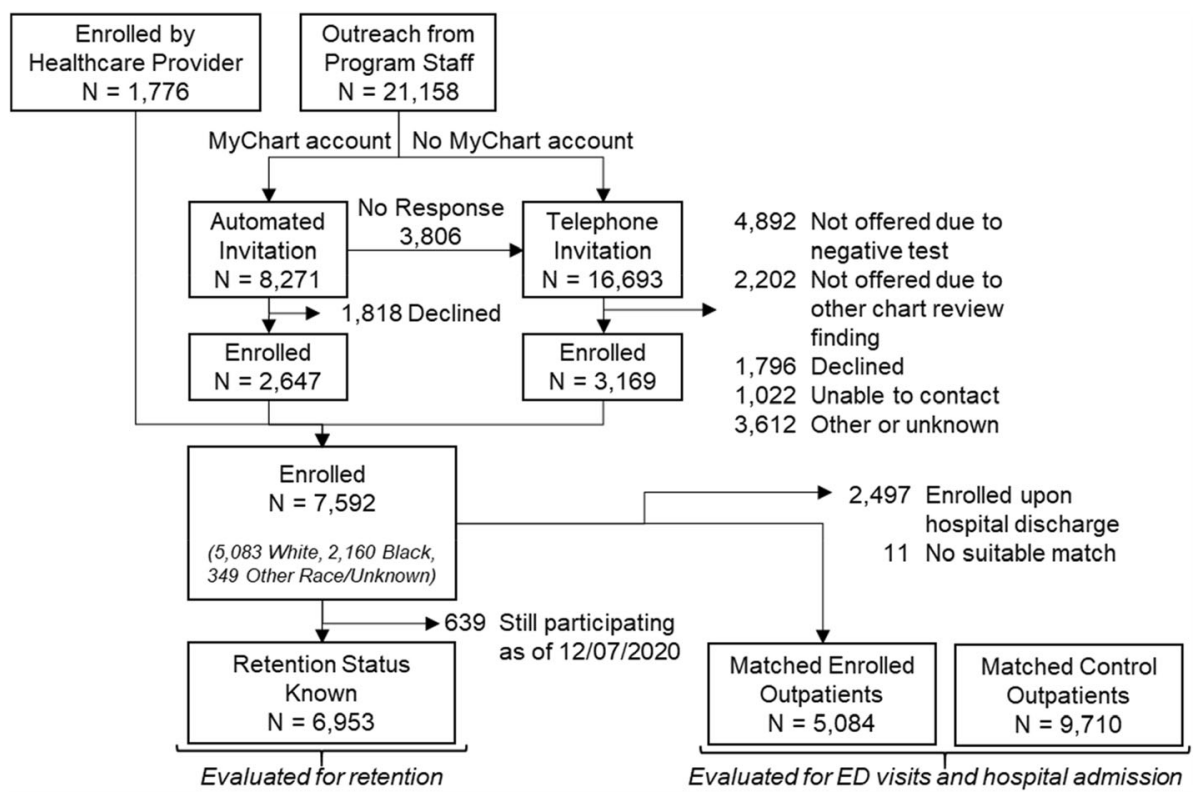

Fig. 1 Flow of patients through the home monitoring program 


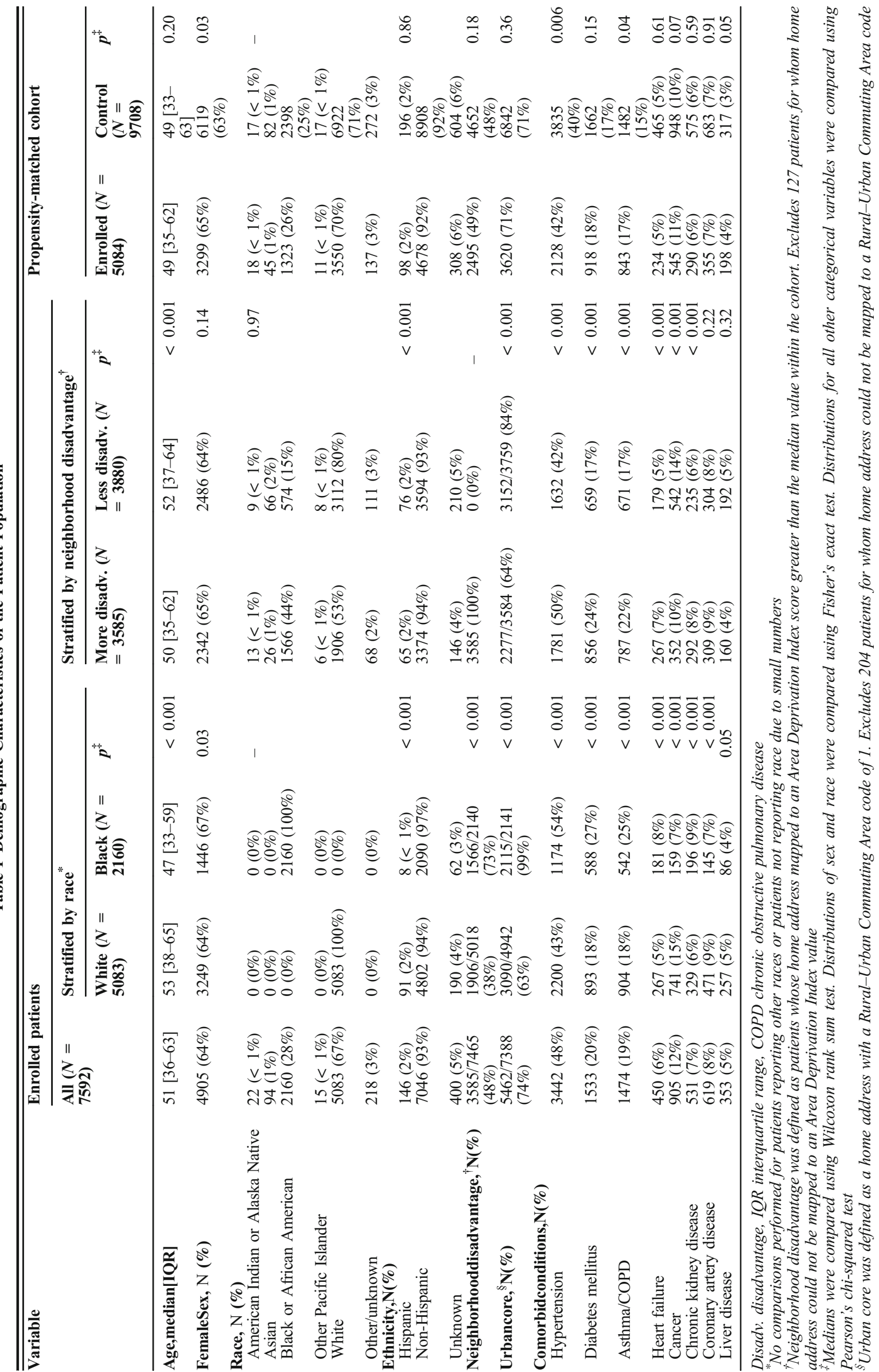


0.009, Table 2). Compared to enrollees from less disadvantaged neighborhoods, enrollees from more disadvantaged neighborhoods were more likely to select the telephone arm ( $59 \%$ versus $43 \%, p=0.02$ ). Enrollees with higher age, male sex, and chronic health conditions were also more likely to select the telephone arm (Supplement Table S2).

A total of 2497 enrollees were excluded from the propensity-matched analysis because their initial COVID test had been performed during a hospital admission, and 11 had no suitable match (Fig. 1). Thus, 5084 enrollees were matched to 9710 COVID-positive outpatient controls. The matched enrollees had a higher frequency of female sex, hypertension, and asthma/COPD than matched controls (Table 1).

\section{Retention of Enrollees}

The enrollees were followed for a median of 8 (IQR 5-11) days (Supplement Figure S2). Enrollees in the app arm required a telephone call due to nonresponse on 4937 of 50,215 patient-days (10\%). Excluding the 639 enrollees still actively participating at the time of data collection, 4337 of 6953 enrollees $(62 \%)$ stayed active in the program until symptom resolution or hospital admission. Compared to White enrollees, Black enrollees had similar rates of retention $(63 \%$ versus $62 \%, p=0.76$, Table 2 ). These findings were driven by differential retention by program arm: Black enrollees had lower retention than white enrollees in the app arm $(49 \%$ versus $55 \%, p=0.01)$ but not in the telephone arm $(69 \%$ versus $71 \%, p=0.12$ ). Enrollees from more versus less disadvantaged neighborhoods had similar retention, both in the program overall and within each program arm (Table 2).

\section{ED Visits and Hospital Admissions in the Propensity-Matched Cohort}

Of the 5084 enrollees included in the propensity-matched analysis, 708 (14\%) had an ED visit and 338 (7\%) had a hospital admission within 30 days. Five hundred forty-eight of these ED visits (77\%) and 259 of these hospital admissions (77\%) occurred without a preceding escalation to a home monitoring triage nurse. Of the 9710 controls, $732(8 \%)$ had an ED visit and 505 (5\%) had a hospital admission within 30 days. Enrollees were more likely to visit the ED (hazard ratio $1.70,95 \%$ CI $1.56-1.87$ ) and be admitted to the hospital (hazard ratio 1.16, 95\% CI 1.02-1.31) compared to matched controls (Fig. 2). The ED visit results were similar in all race and neighborhood advantage groups, with larger effect sizes in the telephone arm than in the app arm (Table 3). The increase in hospital admissions was not observed in the app arm, nor was it observed in White enrollees or those from less disadvantaged neighborhoods.

\section{DISCUSSION}

We have described the implementation of a home monitoring program for COVID-positive patients offering two options for patient engagement (app and telephone), and we assessed its patient engagement characteristics and impact on ED and hospital utilization. Black enrollees had lower program retention rates than white enrollees in the app arm, but not in the telephone arm or in the program as a whole. Enrollees from more and less disadvantaged neighborhoods had similar retention rates. Program enrollees were more likely to visit the ED and be admitted to the hospital than propensity-matched controls.

Even though Black patients had lower retention than White patients in the app arm, preferential selection of the telephone arm by Black enrollees caused there to be no difference in retention across racial strata in the program as a whole (Table 2). Although we do not know what would have happened if the telephone arm had been unavailable, its presence appears to have bridged an engagement gap for Black patients. Failure to bridge this gap might have worsened disparities

Table 2 Patient Engagement Measures Among Enrollees

\begin{tabular}{|c|c|c|c|c|c|c|c|}
\hline \multirow[t]{2}{*}{ Variable } & \multirow{2}{*}{$\begin{array}{l}\text { All enrolled patients }(N= \\
7592)\end{array}$} & \multicolumn{3}{|l|}{ By race ${ }^{*}$} & \multicolumn{3}{|c|}{ By neighborhood disadvantage ${ }^{\dagger}$} \\
\hline & & $\begin{array}{l}\text { White } \\
(N=5083)\end{array}$ & $\begin{array}{l}\text { Black } \\
(N=2160)\end{array}$ & $p$ & $\begin{array}{l}\text { Less disadv. } \\
(N=3585)\end{array}$ & $\begin{array}{l}\text { More disadv. } \\
(N=\mathbf{3 8 8 0})\end{array}$ & $p$ \\
\hline \multicolumn{8}{|c|}{ Programarmselection } \\
\hline Telephone arm & $3899(51 \%)$ & $2224(44 \%)$ & $1471(68 \%)$ & 0.009 & $1673(43 \%)$ & $2132(59 \%)$ & 0.02 \\
\hline App arm & $3693(49 \%)$ & $2859(56 \%)$ & $689(32 \%)$ & - & $2207(57 \%)$ & $1453(41 \%)$ & - \\
\hline \multicolumn{8}{|l|}{ Retention } \\
\hline Whole & $4337 / 6953(62 \%)$ & $2872 / 4621$ & $1264 / 2020$ & 0.76 & $2167 / 3515$ & $2075 / 3315$ & 0.44 \\
\hline program & & $(62 \%)$ & $(63 \%)$ & & $(62 \%)$ & $(63 \%)$ & \\
\hline Telephone arm & $2555 / 3636(70 \%)$ & $\begin{array}{l}1467 / 2063 \\
(71 \%)\end{array}$ & $951 / 1385(69 \%)$ & 0.12 & $\begin{array}{l}1110 / 1551 \\
(72 \%)\end{array}$ & $\begin{array}{l}1369 / 1993 \\
(69 \%)\end{array}$ & 0.06 \\
\hline App arm & $1782 / 3317(54 \%)$ & $\begin{array}{l}1405 / 2558 \\
(55 \%)\end{array}$ & $313 / 635(49 \%)$ & 0.01 & $\begin{array}{l}1057 / 1964 \\
(54 \%)\end{array}$ & $706 / 1322(53 \%)$ & 0.79 \\
\hline
\end{tabular}

Disadv. disadvantage

"No comparisons performed for patients reporting other races or patients not reporting race due to small numbers

${ }^{\dagger}$ Neighborhood disadvantage was defined as patients whose home address mapped to an Area Deprivation Index score greater than the median value within the cohort. Excludes 127 patients for whom home address could not be mapped to an Area Deprivation Index value

${ }^{7}$ Defined as continued participation until symptom resolution (or 14 days if asymptomatic) or until hospital admission. Enrollees who were still participating in the program at the time of the data collection are excluded 

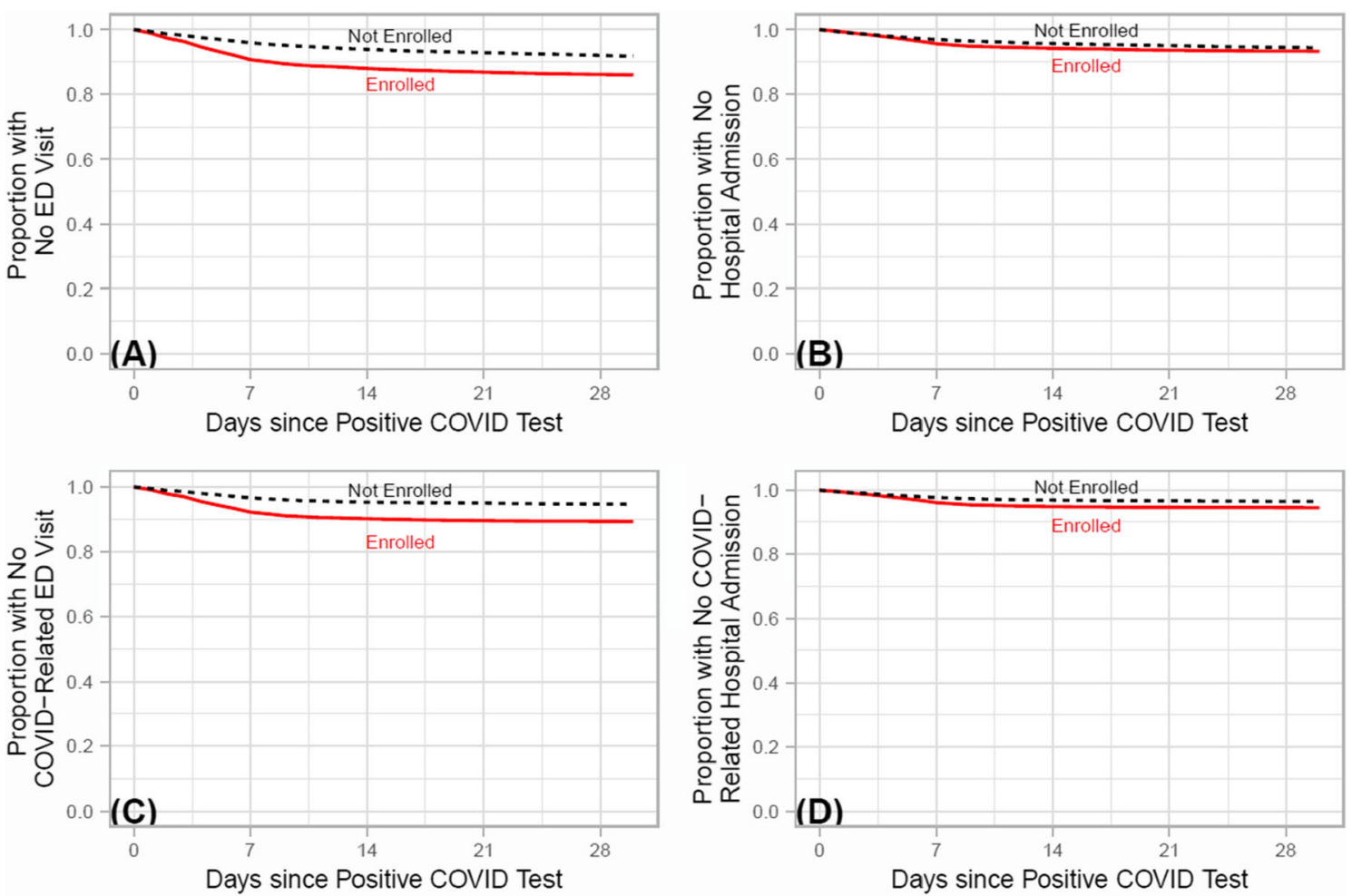

Fig. 2 Time from the date of the patient's positive COVID test until first emergency department (ED) visit (A), hospital admission (B), COVIDrelated ED visit (C), or COVID-related hospital admission (D) among ambulatory patients enrolled in the home monitoring program and a propensity-matched cohort of COVID-positive ambulatory patients not enrolled in the home monitoring program

between Black patients and other patients that already exist due to structural injustices (e.g., housing discrimination, limited public transportation) that limit access to primary care, nutritious food, and exercise. ${ }^{23-26}$ We did not ask patients why they chose one program arm over the other, so we do not know if limited access to smart devices, limited internet connectivity, data privacy concerns, ${ }^{27,28}$ or other factors impacted their choices. Distrust in healthcare organizations that have mistreated Black patients in the past may contribute to the engagement gap. ${ }^{29,30}$ Our findings suggest that home monitoring programs may need to offer non-app-based options for participation to ensure they reach all groups of patients.

Enrollees in our home monitoring program were more likely to visit the ED or be admitted to the hospital than propensity-matched controls. This finding may illustrate the role of the home monitoring program in identifying patients who are deteriorating and directing them to seek care. However, the majority of ED visits and hospital admissions were patient-initiated or provider-initiated without following the formal escalation pathway through the home monitoring program. This may mean the criteria for triggering an escalation need to be loosened so the monitoring program becomes more sensitive, or it may mean patients did not complete the daily questionnaire on days when they felt worse because they already had alternative access to care.

The experiences of a few other groups offering remote monitoring to patients with COVID-19 provide context for our findings. A group from Northwestern Medicine used an EHR portal-based survey to track patient symptoms, with escalation to phone calls if the patient reported concerning symptoms or did not respond to the survey. ${ }^{31}$ Patient nonresponse was reported on about $20 \%$ of patient-days, which is higher than the daily nonresponse rates in our app arm. One explanation is that in our program, patients who were more likely to become nonresponders may have been more likely to choose the telephone option from the start. Enhanced retention using telephone outreach may partly be due to patients' desire to have human interaction while on quarantine - multiple participants reported this to be the best part of their experience. The University of Pennsylvania implemented a monitoring program using automated text messages, with phone calls from nurses to patients who reported worsening symptoms. ${ }^{32}$ Of the first 3000 invited patients, $78 \%$ participated in the program. This adoption rate is higher than in our study, possibly because invitations were only sent to patients with cellular phones. In addition, it is unclear whether clinicians discussed the home monitoring program with patients prior to sending invitations, which would artificially increase the reported adoption rate if invitations were only sent to patients who had verbally expressed interest. ${ }^{32}$ Similar to our findings, the majority of ED visits occurred outside of the program's escalation pathway. In contrast to our findings, participants in a COVID home monitoring program at the Cleveland Clinic had fewer hospitalizations but more outpatient visits compared to non-participants. ${ }^{33}$ The different results may be explained by variability in the program escalation pathways 
Table 3 Propensity-Matched Analysis of Emergency Department Visits and Hospital Admissions

\begin{tabular}{|c|c|c|c|c|c|c|c|c|c|}
\hline \multirow[t]{2}{*}{ Variable } & \multicolumn{3}{|c|}{ Whole program } & \multicolumn{3}{|c|}{ Telephone arm } & \multicolumn{3}{|c|}{ App arm } \\
\hline & Enr & Ctrl & $\begin{array}{l}\text { Hazard ratio } \\
(95 \% \text { CI })\end{array}$ & Enr & Ctrl & $\begin{array}{l}\text { Hazard ratio } \\
(95 \% \text { CI) }\end{array}$ & Enr & Ctrl & Hazard ratio $(95 \% \mathrm{CI})$ \\
\hline \multicolumn{10}{|l|}{ Allpatients } \\
\hline & 5084 & 9710 & & 2431 & 4619 & & 2653 & 5091 & \\
\hline ED visit & $\begin{array}{l}708 \\
(14 \%)\end{array}$ & $\begin{array}{l}732 \\
(8 \%)\end{array}$ & $1.70(1.56-1.87)$ & $\begin{array}{l}454 \\
(19 \%)\end{array}$ & $\begin{array}{l}392 \\
(8 \%)\end{array}$ & $2.00(1.78-2.25)$ & $\begin{array}{l}254 \\
(10 \%)\end{array}$ & $\begin{array}{l}340 \\
(7 \%)\end{array}$ & $1.31(1.14-1.52)$ \\
\hline $\begin{array}{l}\text { COVID- } \\
\text { related ED } \\
\text { visit }\end{array}$ & $\begin{array}{l}541 \\
(11 \%)\end{array}$ & $\begin{array}{l}491 \\
(5 \%)\end{array}$ & $2.01(1.79-2.25)$ & $\begin{array}{l}352 \\
(14 \%)\end{array}$ & $\begin{array}{l}262 \\
(6 \%)\end{array}$ & $2.47(2.13-2.87)$ & $\begin{array}{l}189 \\
(7 \%)\end{array}$ & $\begin{array}{l}229 \\
(4 \%)\end{array}$ & $1.46(1.21-1.75)$ \\
\hline Hospital & 338 & 505 & $1.16(1.02-1.31)$ & 233 & 284 & $1.36(1.17-1.59)$ & 105 & 221 & $0.89(0.73-1.09)$ \\
\hline admission & $(7 \%)$ & $(5 \%)$ & & $(10 \%)$ & $(6 \%)$ & & $(4 \%)$ & $(4 \%)$ & \\
\hline $\begin{array}{l}\text { COVID- } \\
\text { related ad- } \\
\text { mission }\end{array}$ & $\begin{array}{l}278 \\
(5 \%)\end{array}$ & $\begin{array}{l}322 \\
(3 \%)\end{array}$ & $1.46(1.26-1.70)$ & $\begin{array}{l}198 \\
(8 \%)\end{array}$ & $\begin{array}{l}184 \\
(4 \%)\end{array}$ & $1.77(1.47-2.14)$ & $\begin{array}{l}80 \\
(3 \%)\end{array}$ & $\begin{array}{l}138 \\
(3 \%)\end{array}$ & $1.00(0.77-1.31)$ \\
\hline \multicolumn{10}{|l|}{ White } \\
\hline$N$ & 3550 & 6922 & & 1415 & 2760 & & 2135 & 4162 & \\
\hline ED visit & $\begin{array}{l}421 \\
(12 \%)\end{array}$ & $\begin{array}{l}460 \\
(7 \%)\end{array}$ & $1.68(1.49-1.90)$ & $\begin{array}{l}245 \\
(17 \%)\end{array}$ & $\begin{array}{l}199 \\
(7 \%)\end{array}$ & $2.32(1.95-2.76)$ & $\begin{array}{l}176 \\
(8 \%)\end{array}$ & $\begin{array}{l}261 \\
(6 \%)\end{array}$ & $1.21(1.01-1.45)$ \\
\hline $\begin{array}{l}\text { COVID- } \\
\text { related ED } \\
\text { visit }\end{array}$ & $\begin{array}{l}342 \\
(10 \%)\end{array}$ & $\begin{array}{l}338 \\
(5 \%)\end{array}$ & $1.84(1.59-2.14)$ & $\begin{array}{l}205 \\
(14 \%)\end{array}$ & $\begin{array}{l}152 \\
(6 \%)\end{array}$ & $2.48(2.03-3.04)$ & $\begin{array}{l}137 \\
(6 \%)\end{array}$ & $\begin{array}{l}186 \\
(4 \%)\end{array}$ & $1.32(1.06-1.64)$ \\
\hline Hospital & 215 & 363 & $1.04(0.89-1.22)$ & 141 & 172 & $1.39(1.13-1.71)$ & 74 & 191 & $0.74(0.58-0.94)$ \\
\hline $\begin{array}{l}\text { admission } \\
\text { COVID- }\end{array}$ & $(6 \%)$ & $(5 \%)$ & & $(10 \%)$ & $(6 \%)$ & & $(3 \%)$ & $(5 \%)$ & \\
\hline $\begin{array}{l}\text { COVID- } \\
\text { mission }\end{array}$ & $\begin{array}{l}179 \\
(5 \%)\end{array}$ & $\begin{array}{l}236 \\
(3 \%)\end{array}$ & $1.32(1.09-1.59)$ & $\begin{array}{l}123 \\
(9 \%)\end{array}$ & $\begin{array}{l}115 \\
(4 \%)\end{array}$ & $1.84(1.44-2.35)$ & $\begin{array}{l}56 \\
(3 \%)\end{array}$ & $\begin{array}{l}121 \\
(3 \%)\end{array}$ & $0.81(0.59-1.10)$ \\
\hline \multicolumn{10}{|l|}{ Black } \\
\hline$N$ & 1323 & 2400 & & 906 & 1660 & & 417 & 740 & \\
\hline ED visit & $\begin{array}{l}272 \\
(21 \%)\end{array}$ & $\begin{array}{l}259 \\
(11 \%)\end{array}$ & $1.69(1.48-1.94)$ & $\begin{array}{l}200 \\
(22 \%)\end{array}$ & $\begin{array}{l}185 \\
(11 \%)\end{array}$ & $1.80(1.53-2.11)$ & $\begin{array}{l}72 \\
(17 \%)\end{array}$ & $\begin{array}{l}74 \\
(10 \%)\end{array}$ & $1.44(1.11-1.88)$ \\
\hline $\begin{array}{l}\text { COVID- } \\
\text { related ED } \\
\text { visit }\end{array}$ & $\begin{array}{l}186 \\
(14 \%)\end{array}$ & $\begin{array}{l}143 \\
(6 \%)\end{array}$ & $2.30(1.88-2.80)$ & $\begin{array}{l}140 \\
(15 \%)\end{array}$ & $\begin{array}{l}102 \\
(6 \%)\end{array}$ & $2.55(2.03-3.22)$ & $\begin{array}{l}46 \\
(11 \%)\end{array}$ & $\begin{array}{l}41 \\
(6 \%)\end{array}$ & $1.73(1.17-2.55)$ \\
\hline $\begin{array}{r}\text { Hospital } \\
\text { admission }\end{array}$ & $\begin{array}{l}114 \\
(9 \%)\end{array}$ & $\begin{array}{l}131 \\
(5 \%)\end{array}$ & $1.37(1.11-1.68)$ & $\begin{array}{l}87 \\
(10 \%)\end{array}$ & $\begin{array}{l}104 \\
(6 \%)\end{array}$ & $1.35(1.07-1.71)$ & $\begin{array}{l}27 \\
(6 \%)\end{array}$ & $\begin{array}{l}27 \\
(4 \%)\end{array}$ & $1.42(0.94-2.15)$ \\
\hline $\begin{array}{l}\text { COVID- } \\
\text { related ad- }\end{array}$ & $\begin{array}{l}93 \\
(7 \%)\end{array}$ & $\begin{array}{l}82 \\
(3 \%)\end{array}$ & $1.78(1.36-2.33)$ & $\begin{array}{l}72 \\
(8 \%)\end{array}$ & $\begin{array}{l}65 \\
(4 \%)\end{array}$ & $1.75(1.3-2.37)$ & $\begin{array}{l}21 \\
(5 \%)\end{array}$ & $\begin{array}{l}17 \\
(2 \%)\end{array}$ & $1.93(1.08-3.47)$ \\
\hline mission & & & & & & & & & \\
\hline \multicolumn{10}{|l|}{ Lessdisadv } \\
\hline$N$ & 2589 & 5058 & & 1004 & 1959 & & 1585 & 3099 & \\
\hline ED visit & $\begin{array}{l}311 \\
(12 \%)\end{array}$ & $\begin{array}{l}330 \\
(7 \%)\end{array}$ & $1.65(1.43-1.90)$ & $\begin{array}{l}172 \\
(17 \%)\end{array}$ & $\begin{array}{l}150 \\
(8 \%)\end{array}$ & $1.95(1.61-2.37)$ & $\begin{array}{l}139 \\
(9 \%)\end{array}$ & $\begin{array}{l}180 \\
(6 \%)\end{array}$ & $1.36(1.11-1.67)$ \\
\hline $\begin{array}{l}\text { COVID- } \\
\text { related ED } \\
\text { visit }\end{array}$ & $\begin{array}{l}247 \\
(10 \%)\end{array}$ & $\begin{array}{l}236 \\
(5 \%)\end{array}$ & $1.93(1.62-2.29)$ & $\begin{array}{l}137 \\
(14 \%)\end{array}$ & $\begin{array}{l}109 \\
(6 \%)\end{array}$ & $2.30(1.81-2.93)$ & $\begin{array}{l}110 \\
(7 \%)\end{array}$ & $\begin{array}{l}127 \\
(4 \%)\end{array}$ & $1.59(1.24-2.04)$ \\
\hline $\begin{array}{l}\text { Hospital } \\
\text { admission }\end{array}$ & $\begin{array}{l}150 \\
(6 \%)\end{array}$ & $\begin{array}{l}239 \\
(5 \%)\end{array}$ & $1.10(0.91-1.32)$ & $\begin{array}{l}93 \\
(9 \%)\end{array}$ & $\begin{array}{l}110 \\
(6 \%)\end{array}$ & $1.39(1.08-1.80)$ & $\begin{array}{l}57 \\
(4 \%)\end{array}$ & $\begin{array}{l}129 \\
(4 \%)\end{array}$ & $0.86(0.65-1.13)$ \\
\hline $\begin{array}{l}\text { COVID- } \\
\text { related ad- }\end{array}$ & $\begin{array}{l}128 \\
(5 \%)\end{array}$ & $\begin{array}{l}152 \\
(3 \%)\end{array}$ & $1.44(1.15-1.80)$ & $\begin{array}{l}83 \\
(8 \%)\end{array}$ & $\begin{array}{l}72 \\
(4 \%)\end{array}$ & $1.90(1.42-2.56)$ & $\begin{array}{l}45 \\
(3 \%)\end{array}$ & $\begin{array}{l}80 \\
(3 \%)\end{array}$ & $0.98(0.69-1.40)$ \\
\hline mission & & & & & & & & & \\
\hline \multicolumn{10}{|l|}{ Moredisadv } \\
\hline & 2495 & 4652 & & 1427 & 2660 & & 1068 & 1992 & \\
\hline ED visit & $\begin{array}{l}397 \\
(16 \%)\end{array}$ & $\begin{array}{l}402 \\
(9 \%)\end{array}$ & $1.71(1.52-1.93)$ & $\begin{array}{l}282 \\
(20 \%)\end{array}$ & $\begin{array}{l}242 \\
(9 \%)\end{array}$ & $2.02(1.75-2.33)$ & $\begin{array}{l}115 \\
(11 \%)\end{array}$ & $\begin{array}{l}160 \\
(8 \%)\end{array}$ & $1.23(1.00-1.52)$ \\
\hline $\begin{array}{l}\text { COVID- } \\
\text { related ED } \\
\text { visit }\end{array}$ & $\begin{array}{l}294 \\
(12 \%)\end{array}$ & $\begin{array}{l}255 \\
(5 \%)\end{array}$ & $2.06(1.76-2.41)$ & $\begin{array}{l}215 \\
(15 \%)\end{array}$ & $\begin{array}{l}153 \\
(6 \%)\end{array}$ & $2.58(2.12-3.13)$ & $\begin{array}{l}79 \\
(7 \%)\end{array}$ & $\begin{array}{l}102 \\
(5 \%)\end{array}$ & $1.29(0.97-1.71)$ \\
\hline $\begin{array}{l}\text { Hospital } \\
\text { admission }\end{array}$ & $\begin{array}{l}188 \\
(8 \%)\end{array}$ & $\begin{array}{l}266 \\
(6 \%)\end{array}$ & $1.19(1.02-1.40)$ & $\begin{array}{l}140 \\
(10 \%)\end{array}$ & $\begin{array}{l}174 \\
(7 \%)\end{array}$ & $1.34(1.10-1.62)$ & $\begin{array}{l}48 \\
(4 \%)\end{array}$ & $\begin{array}{l}92 \\
(5 \%)\end{array}$ & $0.93(0.69-1.25)$ \\
\hline $\begin{array}{l}\text { COVID- } \\
\text { related ad- } \\
\text { mission }\end{array}$ & $\begin{array}{l}150 \\
(6 \%)\end{array}$ & $\begin{array}{l}170 \\
(4 \%)\end{array}$ & $1.48(1.20-1.82)$ & $\begin{array}{l}115 \\
(8 \%)\end{array}$ & $\begin{array}{l}112 \\
(4 \%)\end{array}$ & $1.69(1.32-2.15)$ & $\begin{array}{l}35 \\
(3 \%)\end{array}$ & $\begin{array}{l}58 \\
(3 \%)\end{array}$ & $1.04(0.69-1.58)$ \\
\hline
\end{tabular}

Enr. enrolled outpatients, Ctrl. control outpatients, CI confidence interval, ED emergency department, Disadv. disadvantage

(each program may have a different threshold for referring to inpatient versus outpatient care).

This work has limitations. Firstly, reliable measures of vital status were unavailable. This makes it difficult to tell if the higher ED and hospital utilization rates among program participants are preventing deaths that may have occurred if the patients had stayed home. Secondly, no indicator of COVID infection severity was available. Although patients were matched based on demographic characteristics and comorbid conditions, it is possible that patients with more severe symptoms were more likely to enroll in the home monitoring program and subsequently were more likely to require 
advanced care. To address this limitation, we excluded patients whose COVID test was performed during a hospital admission (who presumably had more severe disease). Thirdly, data on ED and hospital utilization were only available within the BJC Healthcare system. Visits to non-affiliated facilities would not have been detected in our analysis. Fourthly, reliable data on program costs were not available. Telephone monitoring requires much more medical assistant time than automated monitoring, driving expenses upward. Complete economic analysis, including estimates of savings from theoretically prevented morbidity, was beyond the scope of this work but could be explored in the future. Fifthly, telephone monitoring requires a large pool of available medical staff. Although we addressed this issue by redeploying staff from locations that had temporarily closed, this need may limit the scalability or repeatability of this program. In fact, additional staff needed to be recruited during the final months of 2020 to accommodate a surge in patient enrollment. Sixthly, this work was conducted within a single healthcare system. Differences in local patient populations might lead to differences in engagement if the same intervention were deployed at other institutions.

In conclusion, a home monitoring program for COVIDpositive outpatients that offered both smart device app-based monitoring and telephone-based monitoring was able to achieve modest adoption and moderate-to-high patient retention, while causing an increase in ED and hospital utilization. Offering the telephone-based option may have made the program more accessible to Black patients, removing one barrier that may affect health outcomes in this population. The lessons learned here can influence the design of remote monitoring interventions for other acute health conditions or at other institutions.

Supplementary Information The online version contains supplementary material available at https://doi.org/10.1007/s11606-02107207-4.

Acknowledgements: Contributors: The authors would like to acknowledge Maria Gerritzen, Stacy Olinger, Nathan Moore, Michele Thomas, Janice Ernest, and Beth LeCorgne for their leadership and support of the COVID Home Monitoring Program and Andrew Guyton for the hours of technical build to make the program work, as well as Julie Fox and the group of medical assistants, care managers, and nurses caring for COVID patients every day. We never tire of hearing the patients praise the tremendous job they do every day.

Corresponding Author: Bradley A. Fritz, MD, MSc; Department of Anesthesiology, Washington University School of Medicine, St. Louis, MO, USA (e-mail: bafritz@wustl.edu).

Funding This work was supported by BJC Healthcare and Washington University School of Medicine. Dr. Fritz received support from the National Institutes of Health (T32 GM108539) and the Foundation for Anesthesia Education and Research (MRTG08152020FRITZ) during the conduct of this study. Dr. Maddox discloses current grant funding from the NIH NCATS (1U24TR002306-01: A National Center for Digital Health Informatics Innovation) and honoraria and/or expense reimbursement in the past 3 years from the Henry Ford health system (March 2019), the University of California San Diego (January 2020), the University of Chicago (Jan 2021) and George Washington University (Jan 2021). He has also received travel expense reimbursement for meetings connected to the American College of Cardiology. He is currently employed as a cardiologist and the executive director of the Healthcare Innovation Lab at BJC HealthCare/Washington University School of Medicine. In this capacity, he is advising Myia Labs, for which his employer is receiving equity compensation in the company. He is receiving no individual compensation from the company. He is also a compensated director for a New Mexico-based foundation, the J.F Maddox Foundation.

\section{Declarations:}

Conflict of Interest: The authors declare that they have no conflicts of interest.

\section{REFERENCES}

1. GandhiRT,LynchJB,delRioC. Mild or moderate COVID-19. N Engl J Med. 2020;383:1757-66.

2. Gargs,KimL, WhitakerM,etal. Hospitalization rates and characteristics of patients hospitalized with laboratory-confirmed coronavirus disease 2019-COVID-NET, 14 States, March 1-30, 2020. MMWR Morb Mortal Wkly Rep. 2020;69:458-64.

3. ZhouM,ZhangX,QuJ. Coronavirus disease 2019 (COVID-19): a clinical update. Front Med. 2020;14:126-35.

4. HuangC,WangY,LiX,etal. Clinical features of patients infected with 2019 novel coronavirus in Wuhan, China. Lancet. 2020;395:497-506.

5. MoghadasSM,ShoukatA,FitzpatrickMC,etal. Projecting hospital utilization during the COVID-19 outbreaks in the United States. Proc Natl Acad Sci U S A. 2020;117:9122-6.

6. HollanderJE,CarrBG. Virtually perfect? Telemedicine for COVID-19. N Engl J Med. 2020;382:1679-81.

7. NelsonR. Telemedicine and telehealth: the potential to improve rural access to care. Am J Nurs. 2017;117:17-8.

8. SyedST,GerberBS,SharpLK. Traveling towards disease: transportation barriers to health care access. J Community Health. 2013;38:976-93.

9. ChoiY,NamK,KimCY. Association between convenience of transportation and unmet healthcare needs of rural elderly in Korea. J Prev Med Public Health. 2019;52:355-65.

10. AndersenTO,NielsenKD,MollJ,SvendsenJH. Unpacking telemonitoring work: Workload and telephone calls to patients in implanted cardiac device care. Int J Med Inform. 2019;129:381-7.

11. MooneyKH,BeckSL,WongB,etal. Automated home monitoring and management of patient-reported symptoms during chemotherapy: results of the symptom care at home RCT. Cancer Med. 2017;6:537-46.

12. PersellSD,PeprahYA,LipiszkoD,etal. Effect of home blood pressure monitoring via a smartphone hypertension coaching application or tracking application on adults with uncontrolled hypertension: a randomized clinical trial. JAMA Netw Open. 2020;3:e200255.

13. CullenR. Addressing the digital divide. Online Inf Rev. 2001;25:311-20.

14. RamsettyA,AdamsC. Impact of the digital divide in the age of COVID-19. J Am Med Inform Assoc. 2020;27:1147-8.

15. MishoriR,AntonoB. Telehealth, rural America, and the digital divide. J Ambul Care Manage. 2020;43:319-22.

16. Lai J, Widmar NO. Revisiting the digital divide in the COVID-19 era. Appl Econ Perspect Policy. 2021;43:458-64.

17. Home monitoring for COVID-19 now available through MyChart: Cleveland Clinic and Epic introduce new functionality. Consult $Q D$ 2020; https://consultqd.clevelandclinic.org/home-monitoring-for-covid-19now-available-through-mychart/. Accessed February 10, 2021.

18. MedinaM,BabiuchC,CardM,etal. Home monitoring for COVID-19. Cleve Clin J Med. 2020

19. vonElmE,AltmanDG,EggerM,PocockSJ,GotzschePC,VandenbrouckeJP. Strengthening the Reporting of Observational Studies in Epidemiology (STROBE) statement: guidelines for reporting observational studies. BMJ (Clin Res Ed.). 2007;335:806-8.

20. KindAJH,BuckinghamWR. Making neighborhood-disadvantage metrics accessible - The Neighborhood Atlas. N Engl J Med. 2018;378:2456-8.

21. OnegaT,WeissJE,Alford-TeasterJ,GoodrichM,EliassenMS,KimSJ. Concordance of rural-urban self-identity and zip code-derived rural-urban commuting area (RUCA) designation. J Rural Health. 2020;36:274-80.

22. StuartEA,KingG,Imaik,HoD. MatchIt: nonparametric preprocessing for parametric causal inference. J Stat Softw. 2011;42:1-28. 
23. RollstonR,Galeas. COVID-19 and the social determinants of health. Am J Health Promot. 2020;34:687-9.

24. KarmakarM,LantzPM,TipirneniR. Association of social and demographic factors with COVID-19 incidence and death rates in the US. JAMA Netw Open. 2021;4:e2036462.

25. BergerZ,AltieryDEJV,AssoumouSA,GreenhalghT. Long COVID and health inequities: the role of primary care. Milbank Q. 2021;99:519-41.

26. BaileyZD,KriegerN,AgénorM,GravesJ,LinosN,BassettMT. Structural racism and health inequities in the USA: evidence and interventions. Lancet. 2017;389:1453-63.

27. GalvinHK,DeMuroPR. Developments in privacy and data ownership in mobile health technologies, 2016-2019. Yearb Med Inform. 2020;29:32-43.

28. ZhangB,KrepsS,McMurryN,McCainRM. Americans' perceptions of privacy and surveillance in the COVID-19 pandemic. PLoS One. 2020;15:e0242652.

29. BenJ,CormackD,HarrisR,ParadiesY. Racism and health service utilisation: a systematic review and meta-analysis. PLoS One. 2017;12:e0189900.
30. DehonE,WeissN,JonesJ,FaulconerW,HintonE,Sterlings. A systematic review of the impact of physician implicit racial bias on clinical decision making. Acad Emerg Med. 2017;24:895-904.

31. KrickeG,RoemerPE,DevinP,HenschenBruceL,BiermanJenniferA,LinderJeffreyA. Rapid implementation of an outpatient Covid-19 monitoring program. NEJM Catal Innov Care Deliv. 2020.

32. MorganAU,BalachandranM,DoD,etal. Remote monitoring of patients with Covid-19: design, implementation, and outcomes of the first 3,000 patients in COVID Watch. NEJM Catal Innov Care Deliv. 2020.

33. Misra-HebertAD,JiX,JehiL,etal. COVID-19 home monitoring after diagnosis and health care utilization in an integrated health system. JAMA Health Forum. 2021;2:e210333-e.

Publisher's Note Springer Nature remains neutral with regard to jurisdictional claims in published maps and institutional affiliations. 